\title{
The predictive and prognostic values of factors associated with visceral pleural involvement in resected lung adenocarcinomas
}

This article was published in the following Dove Press journal:

OncoTargets and Therapy

20 April 2016

Number of times this article has been viewed

\author{
Huibiao Zhang' \\ Chen Lu ${ }^{2}$ \\ Yingjie Lu' \\ Bo Yu ${ }^{2}$ \\ Fanzhen Lv' \\ Zhenghong Zhu' \\ 'Department of Thoracic Surgery, \\ ${ }^{2}$ Department of Pathology, Huadong \\ Hospital, Fudan University, Shanghai, \\ People's Republic of China
}

Correspondence: Zhenghong Zhu Department of Thoracic Surgery, Huadong Hospital, Fudan University, 221 West Yan'an Road, Shanghai 200040, People's Republic of China Tel +86 2I 6248 3180; ext 70703 Fax +86 2I 62483180 Email drzhuzhenghong@।26.com
Background: The predictive and prognostic impact of factors associated with visceral pleural invasion (VPI) on survival and recurrence in patients with resected lung adenocarcinomas is not clearly defined.

Patients and methods: A total of 505 consecutive patients with stage Ia-IIIa lung adenocarcinomas treated with radical resection were included. The predominant growth pattern was classified according to the new classification system for lung adenocarcinoma proposed by the International Association for the Study of Lung Cancer, the American Thoracic Society, and the European Respiratory Society. The correlations of VPI with clinical and pathologic parameters were analyzed.

Results: The incidence of VPI was significantly lower in lepidic predominant group (15.5\% vs $4.5 \%, P<0.001)$ and higher in solid and micropapillary predominant group $(28.6 \%$ vs $17.6 \%$, $P=0.004$ and $14.7 \%$ vs $4.2 \%, P<0.001$, respectively). VPI correlated with higher risk in regional postoperative recurrence (hazard ratio, 2.341; 95\% confidence interval, 1.564-3.504) and distant recurrence (hazard ratio, 2.193; 95\% confidence interval, 1.665-2.89) in surgically resected lung adenocarcinomas. However, when growth patterns of adenocarcinoma were lumped into multivariate analysis, VPI was not a significant independent predictive factor for survival ( $P=0.854$ for overall survival $[\mathrm{OS}]$ and $P=0.575$ for disease-free survival [DFS] ) and recurrence ( $P=0.38$ for regional recurrence and $P=0.089$ for distant recurrence). Of the 95 patients with stage Ib, those who received adjuvant chemotherapy had longer DFS and OS than the patients who received no chemotherapy after surgery. However, these differences in DFS and OS did not reach statistical significance ( $P=0.063$ for DFS, $P=0.85$ for OS).

Conclusion: VPI was associated with solid and micropapillary histology. In addition, stage Ib patients with solid histologic subtype tumor showed longer DFS and OS, highlighting a potential benefit in this subgroup of patients and necessitating the need for larger clinical trials.

Keywords: lung cancer, visceral pleural invasion, histologic subtype, TNM stage, follow-up

\section{Introduction}

Lung cancer is the leading cause of cancer-related deaths worldwide. ${ }^{1}$ Visceral pleural invasion (VPI) has been shown as an independent prognostic factor in patients with non-small-cell lung cancers (NSCLCs). ${ }^{2-4}$ Consequently, VPI status was included in the seventh TNM staging system, alongside tumor size. ${ }^{5}$ However, results of some studies have challenged the role of VPI as a prognostic parameter in NSCLCs. ${ }^{6-8}$ Since the existence of VPI upgrades T stage of primary tumor from T1 to T2 in TNM staging system and may determine whether adjuvant chemotherapy is required, it is necessary 
to confirm the prognostic significance and identify the subset of patients with VPI who might benefit from adjuvant chemotherapy.

Adenocarcinoma is the most common pathologic type of NSCLC. In 2011, a new histologic classification system for lung adenocarcinoma was proposed by the International Association for the Study of Lung Cancer (IASLC), the American Thoracic Society (ATS), and the European Respiratory Society (ERS), based on the semiquantitative identification of the predominant histologic subtype. ${ }^{9}$ The IASLC/ATS/ERS classification system is independently associated with overall survival (OS) and disease recurrence in adenocarcinomas. ${ }^{10}$ Subtypes with micropapillary and solid predominant growth patterns have aggressive biological behavior that is associated with lymph node and distant metastasis. ${ }^{10,11}$ It is unclear whether pleural invasion is associated with these aggressive lung adenocarcinoma subtypes.

In the current study, we focused on the VPI status and histologic subtypes based on the new classification system of adenocarcinoma, as well as the possibility of VPI status as an independent predictor for treatment outcomes, including disease-free survival (DFS), OS, and regional and distant failure.

\section{Methods}

\section{Patient selection}

This study was approved by the Institutional Review Board at Fudan University Shanghai Huadong Hospital. From January 2007 to June 2010, a total of 505 consecutive patients who received surgical resection for lung adenocarcinomas were included. Patients with distant metastases, and receiving neoadjuvant chemotherapy, EGFR tyrosine kinase inhibitor, or radiation therapy were ineligible for enrollment. Written informed consent was obtained from all patients before surgery. Clinicopathologic characteristics, including sex, age at diagnosis, pathological TNM stage, tumor differentiation, pleural involvement, and histologic subtype, were collected. Pathologic staging was conducted according to the seventh edition of TNM classification. ${ }^{5}$

\section{Histologic evaluation}

Hematoxylin and eosin (H\&E)-stained slides for each tumor were reviewed independently by two pathologists (CL and BY). Discrepancies were resolved by simultaneous rereview of the slides by both pathologists. In accordance with the IASLC, ATS, and ERS classification of lung adenocarcinoma, ${ }^{9}$ each tumor was classified according to the most dominant pattern (lepidic, acinar, papillary, micropapillary, solid, and invasive mucinous adenocarcinoma).

VPI was defined according to the IASLC criteria: PL0, tumor without pleural involvement beyond its elastic layer; PL1, tumor that invades beyond the elastic layer; and PL2, tumor that invades to the pleural surface. ${ }^{12}$ PL1 and PL2 were defined as VPI. As the IASLC suggested, in cases where it was difficult to determine the presence of VPI by H\&E slides, an elastic stain (Verhoeff's elastic van Gieson) was used.

\section{Statistical analysis}

The $\chi^{2}$ test for categorical variables was used to compare patients' characteristics between patients with VPI and without VPI. Survival curves were estimated using the Kaplan-Meier method. The log-rank test was used to compare survival of groups. Univariable Cox proportional hazards regression models were used to investigate the correlation of selected variables with survival. Multivariable Cox regression analysis was used to assess the correlation of VPI with survival. The two-sided significance level was set at $P<0.05$. Data were analyzed using Statistical Package for the Social Sciences Version 16.0 Software (SPSS Inc., Chicago, IL, USA).

\section{Results}

\section{Patient characteristics}

Patient characteristics are listed in Table 1. There were 272 women and 233 men, with a median age of 57 years (range: 24-83 years). VPI occurred in 266 cases $(52.7 \%)$. The distribution of stages of lung adenocarcinoma with VPI among the patients was: 95 patients (35.7\%), stage Ib; 25 patients (9.4\%), stage IIa; 26 patients (9.8\%), stage IIb; and 120 patients $(45.1 \%)$, stage IIIa.

\section{Correlation between VPI and clinicopathologic characteristics}

The clinical and pathologic characteristics of patients with and without VPI are shown in Table 1. The percentage of female patients was significantly higher in patients with VPI than in those without $(P=0.026)$. Compared with VPI negativity, VPI positivity was associated with a higher frequency of $\mathrm{N} 2$ involvement $(P=0.005)$. The percentage of tumor size $3 \mathrm{~cm}$ or smaller was significantly higher in VPI-positive group $(P<0.001)$. There was no significant difference in the distribution of age $(P=0.592)$, smoking status $(P=0.142)$, tumor differentiation $(P=0.158)$, tumor location $(P=0.665)$, or surgical procedure $(P=0.129)$. 
Table I Baseline characteristics of the study subjects according to visceral pleural involvement

\begin{tabular}{|c|c|c|c|c|c|c|}
\hline \multirow[t]{2}{*}{ Characteristics } & \multirow[t]{2}{*}{ Patients (n) } & \multicolumn{2}{|c|}{ VPI+ subjects $(\mathrm{N}=266)$} & \multicolumn{2}{|c|}{ VPI- patients $(\mathrm{N}=239)$} & \multirow[t]{2}{*}{$P$-value } \\
\hline & & Number & $\%$ & Number & $\%$ & \\
\hline \multicolumn{7}{|l|}{ Sex } \\
\hline Female & 272 & 156 & 58.6 & 116 & 48.5 & \\
\hline Male & 233 & 110 & 41.4 & 123 & 51.5 & 0.026 \\
\hline \multicolumn{7}{|l|}{ Age (years) } \\
\hline$\geq 60$ & 215 & 117 & 44 & 98 & 41 & \\
\hline$<60$ & 290 & 149 & 56 & $|4|$ & 59 & 0.529 \\
\hline \multicolumn{7}{|l|}{ Smoking status } \\
\hline Never smoker & 317 & 175 & 65.8 & 142 & 59.4 & \\
\hline Smoker & 188 & 91 & 34.2 & 97 & 40.6 & 0.142 \\
\hline \multicolumn{7}{|l|}{ Tumor size (cm) } \\
\hline$>3$ & 160 & 53 & 22.2 & 107 & 40.2 & \\
\hline$\leq 3$ & 345 & 186 & 77.8 & 159 & 59.8 & $<0.000$ I \\
\hline \multicolumn{7}{|l|}{ Lymph nodes } \\
\hline No & 281 & 133 & 50 & 148 & 61.9 & \\
\hline $\mathrm{NI}$ & 66 & 33 & 12.4 & 33 & 13.8 & \\
\hline N2 & 158 & 100 & 37.6 & 58 & 24.3 & 0.005 \\
\hline \multicolumn{7}{|l|}{ Stage } \\
\hline la & 113 & 0 & 0 & 113 & 47.3 & \\
\hline $\mathrm{lb}$ & 108 & 95 & 35.7 & 13 & 5.4 & \\
\hline Ila & 49 & 25 & 9.4 & 24 & 10 & \\
\hline Ilb & 32 & 26 & 9.8 & 6 & 2.5 & \\
\hline Illa & 203 & 120 & 45.2 & 83 & 34.7 & $<0.000$ I \\
\hline \multicolumn{7}{|l|}{ Lymphovascular invasion } \\
\hline Positive & 120 & 80 & 30.1 & 40 & 16.7 & \\
\hline Negative & 348 & 169 & 63.5 & 179 & 74.9 & 0.002 \\
\hline \multicolumn{7}{|l|}{ Differentiation } \\
\hline Well differentiated & 83 & 49 & 18.4 & 34 & 14.2 & \\
\hline Moderately differentiated & 280 & 137 & 51.5 & 143 & 59.8 & \\
\hline Poorly differentiated & 142 & 80 & 30.1 & 62 & 25.9 & 0.158 \\
\hline \multicolumn{7}{|l|}{ Initial recurrence site } \\
\hline Local recurrence & 3 & I & 0.40 & 2 & 0.8 & 0.605 \\
\hline Regional recurrence & 80 & 60 & 22.6 & 20 & 8.4 & $<0.000$ I \\
\hline Distant & 165 & 104 & 39.1 & 61 & 25.5 & 0.001 \\
\hline No recurrence & 253 & 101 & 40 & 152 & 63.6 & $<0.000$ I \\
\hline \multicolumn{7}{|l|}{ Location } \\
\hline Right upper lobe & 164 & 80 & 30.1 & 84 & 35.1 & \\
\hline Right middle lobe & 41 & 22 & 8.3 & 19 & 7.9 & \\
\hline Right lower lobe & 85 & 50 & 18.8 & 35 & 14.6 & \\
\hline Left upper lobe & 134 & 72 & 27.1 & 62 & 25.9 & \\
\hline Left lower lobe & 81 & 42 & 15.8 & 39 & 16.3 & 0.665 \\
\hline \multicolumn{7}{|l|}{ Surgical procedure } \\
\hline Wedge & 19 & 6 & 2.3 & 13 & 5.4 & \\
\hline Lobectomy & 436 & 227 & 85.3 & 209 & 87.4 & \\
\hline Bilobectomy & 32 & 21 & 7.9 & 11 & 4.6 & \\
\hline Sleeve lobectomy & 7 & 5 & 1.9 & 2 & 0.8 & \\
\hline Pneumonectomy & 11 & 7 & 2.6 & 4 & 1.7 & 0.129 \\
\hline \multicolumn{7}{|l|}{ Pathologic subtype } \\
\hline Lepidic predominant & 49 & 12 & 4.5 & 37 & 15.5 & $<0.000$ I \\
\hline Acinar predominant & 207 & 104 & 39.1 & 103 & 43.1 & 0.367 \\
\hline Papillary predominant & 53 & 22 & 8.3 & 31 & 13 & 0.109 \\
\hline Solid predominant & 118 & 76 & 28.6 & 42 & 17.6 & 0.004 \\
\hline Micropapillary & 49 & 39 & 14.7 & 10 & 4.2 & $<0.000$ I \\
\hline \multicolumn{7}{|l|}{ predominant } \\
\hline Mucinous predominant & 27 & 13 & 4.9 & 14 & 5.9 & 0.694 \\
\hline
\end{tabular}

Abbreviation: VPI, visceral pleural invasion. 
The percentage of VPI-positive tumors was significantly lower in lepidic predominant group $(15.5 \%$ vs $4.5 \%, P<0.001)$ and higher in solid and micropapillary predominant groups $(28.6 \%$ vs $17.6 \%, P=0.004$ and $14.7 \%$ vs $4.2 \%, P<0.001$, respectively) than that of VPI-negative tumors.

\section{Survival outcome}

The median follow-up time was 43.0 months (mean, 40.2 \pm 17.3 months). Among the 505 patients, 257 (50.9\%) were free of tumor recurrence and 248 (49.1\%) developed recurrence, pathologically confirmed by biopsy in 53\% and clinically diagnosed in $47 \%$ based on imaging assessment. A total of 122 patients died during the follow-up period. The 5-year cumulative incidence of relapse was $46.7 \%$ for all patients. Of 248 patients who had disease relapsed, $121(48.8 \%)$ relapsed within 12 months, 64 (25.8\%) relapsed between 12 and 24 months, and 63 (25.4\%) relapsed 24 months after surgery. The majority of patients had disease relapsed within 12 months after surgery for solid predominant adenocarcinoma (59.5\%, 47/79) and for micropapillary predominant adenocarcinoma $(63.9 \%, 23 / 36)$.

Local recurrence with regional and distant failure occurred in $16.6 \%$ of patients $(3 / 18)$ who received wedge resection. There were 80 patients who had disease recurrence after surgery, with regional failure alone in $10.1 \%$ of 248 patients and both regional and distant failure in $22.2 \%$ of patients (55/248). Distant metastases occurred in 165 patients (66.5\% of 248). Lung was the most common site for distant failure $(n=104)$.

On univariate analysis of clinical and pathologic factors of 505 surgically resected patients for OS and DFS, male, smoking history, advanced $\mathrm{T}$ stage, lymph node metastasis, moderate and poor differentiation, lymphovascular invasion, and VPI were significantly associated with poorer outcome (Table 2).

Patients with lepidic, acinar, papillary, solid, micropapillary, and invasive mucinous adenocarcinoma predominant subtype had 5-year cumulative incidence of relapse of

Table 2 Univariate analyses of associations between the clinical and pathologic characteristics and survival

\begin{tabular}{|c|c|c|c|c|c|}
\hline \multirow[t]{2}{*}{ Variables } & \multirow[t]{2}{*}{ Comparison } & \multicolumn{2}{|l|}{ Overall survival } & \multicolumn{2}{|l|}{ Disease-free survival } \\
\hline & & HR (95\% Cl) & $P$-value & HR (95\% Cl) & $P$-value \\
\hline \multirow[t]{2}{*}{ Age (years) } & $<60$ & 1 & & I & \\
\hline & $\geq 60$ & $0.759(0.526-1.095)$ & 0.14 & $0.836(0.649-1.078)$ & 0.168 \\
\hline \multirow[t]{2}{*}{ Sex } & Female & 1 & & I & \\
\hline & Male & $1.65 \mathrm{I}(1.162-2.347)$ & 0.005 & 1.385 (I.08I-I.773) & 0.01 \\
\hline \multirow[t]{2}{*}{ Smoking status } & Never smoker & 1 & & 1 & \\
\hline & Smoker & $1.72(1.211-2.443)$ & 0.002 & $1.543(1.201-1.98 I)$ & 0.001 \\
\hline \multirow[t]{5}{*}{ Location } & Right upper lobe & 1 & & 1 & \\
\hline & Right middle lobe & 1.034 (0.557-1.919) & 0.915 & I.058 (0.723-I.55) & 0.771 \\
\hline & Right lower lobe & $0.869(0.5 \mid 3-1.472)$ & 0.603 & $1.126(0.672-1.888)$ & 0.651 \\
\hline & Left upper lobe & 0.917 (0.583-1.443) & 0.707 & $1.028(0.663-1.593)$ & 0.903 \\
\hline & Left lower lobe & $0.697(0.394-1.234)$ & 0.215 & I.I 24 (0.757-I.668) & 0.563 \\
\hline \multirow[t]{5}{*}{ Surgical procedure } & Wedge & 1 & & 1 & \\
\hline & Lobectomy & $2.099(0.517-8.52)$ & 0.3 & $2.204(0.834-4.915)$ & 0.119 \\
\hline & Bilobectomy & 5.474 (I.264-23.7I) & 0.023 & 4.207 (I.604-II.032) & 0.003 \\
\hline & Sleeve lobectomy & $10.498(2.028-54.338)$ & 0.005 & $5.507(1.668-18.179)$ & 0.005 \\
\hline & Pneumonectomy & I5.902 (3.369-75.058) & $<0.0001$ & $6.279(2.05 \mathrm{I}-19.222)$ & 0.001 \\
\hline \multirow[t]{5}{*}{ T stage } & Tla & 1 & & I & \\
\hline & TIb & $0.975(0.343-2.768)$ & 0.962 & $1.104(0.616-1.976)$ & 0.74 \\
\hline & $\mathrm{T} 2 \mathrm{a}$ & 3.75 I (2.045-6.879) & $<0.0001$ & $2.835(1.955-4.112)$ & $<0.0001$ \\
\hline & $\mathrm{T} 2 \mathrm{~b}$ & 10.847 (5.227-22.507) & $<0.0001$ & $6.734(4.093-11.078)$ & $<0.0001$ \\
\hline & T3 & $7.223(2.949-17.692)$ & $<0.0001$ & $4.333(2.285-8.219)$ & $<0.0001$ \\
\hline \multirow[t]{3}{*}{ Pathologic N stage } & No & 1 & & 1 & \\
\hline & $\mathrm{NI}$ & $2.886(1.65-5.048)$ & $<0.0001$ & $2.246(1.527-3.305)$ & $<0.0001$ \\
\hline & N2 & $5.433(3.62-8.154)$ & $<0.0001$ & $4.733(3.593-6.234)$ & $<0.0001$ \\
\hline \multirow[t]{4}{*}{ Histology } & Lepidic predominant & 1 & & 1 & \\
\hline & Acinar predominant & $8.353(1.139-61.278)$ & 0.037 & $2.765(1.439-5.313)$ & 0.002 \\
\hline & Papillary predominant & $6.29(0.757-52.259)$ & 0.089 & $1.957(0.903-4.242)$ & 0.089 \\
\hline & Solid predominant & $39.746(5.507-286.86)$ & $<0.0001$ & $6.276(3.25-12.122)$ & $<0.0001$ \\
\hline
\end{tabular}


Table 2 (Continued)

\begin{tabular}{|c|c|c|c|c|c|}
\hline \multirow[t]{2}{*}{ Variables } & \multirow[t]{2}{*}{ Comparison } & \multicolumn{2}{|l|}{ Overall survival } & \multicolumn{2}{|l|}{ Disease-free survival } \\
\hline & & HR (95\% Cl) & $P$-value & HR (95\% Cl) & $P$-value \\
\hline & Micropapillary predoninant & $30.215(4.05-225.43)$ & 0.001 & 6.667 (3.309-13.474) & $<0.0001$ \\
\hline & Mucinous predominant & $15.604(1.876-129.82)$ & 0.011 & $3.012(1.299-6.982)$ & 0.01 \\
\hline \multirow[t]{3}{*}{ Differentiation } & Well differentiated & 1 & & 1 & \\
\hline & Moderately differentiated & $3.775(1.63-8.744)$ & 0.002 & $2.147(1.411-3.268)$ & $<0.0001$ \\
\hline & Poorly differentiated & $9.331(4.028-21.616)$ & $<0.0001$ & $3.716(2.393-5.77 I)$ & $<0.0001$ \\
\hline \multirow[t]{2}{*}{ Visceral pleural invasion } & Negative & 1 & & I & \\
\hline & Positive & $2.511(1.705-3.697)$ & $<0.0001$ & $2.106(1.622-2.734)$ & $<0.0001$ \\
\hline \multirow[t]{2}{*}{ Lymphovascular space invasion } & Negative & 1 & & I & \\
\hline & Positive & $2.953(2.035-4.286)$ & $<0.0001$ & $2.418(1.847-3.167)$ & $<0.0001$ \\
\hline
\end{tabular}

Abbreviations: $\mathrm{Cl}$, confidence interval; $\mathrm{HR}$, hazard ratio.

$75 \%, 48.6 \%, 61.2 \%, 29.7 \%, 27.9 \%$, and $53 \%$, respectively. Patients with lepidic predominant adenocarcinoma had the most favorable outcome. In contrast, micropapillary and solid predominant adenocarcinomas were associated with worse prognosis (Table 2; Figure S1).

As shown in Table 3, both regional and distant failures were significantly associated with male, smoking history, sleeve lobectomy, advanced T stage (T2a-T3), lymph nodes metastasis (N1-2), solid and micropapillary histology, moderate and poor differentiation, lymphovascular invasion, and VPI. In addition, distant recurrence was also significantly associated with bilobectomy, pneumonectomy, acinar, and mucinous adenocarcinoma. Because the number of patients with local failure was too small, we did not perform univariate analysis to identify the factors that correlated with local recurrence.

Table 3 Univariate analyses of associations between the clinical and pathologic characteristics and tumor recurrence

\begin{tabular}{|c|c|c|c|c|c|}
\hline \multirow[t]{2}{*}{ Variables } & \multirow[t]{2}{*}{ Comparison } & \multicolumn{2}{|c|}{ Regional disease-free survival } & \multicolumn{2}{|c|}{ Distant disease-free survival } \\
\hline & & HR (95\% CI) & $\overline{P \text {-value }}$ & HR (95\% Cl) & $P$-value \\
\hline \multirow[t]{2}{*}{ Age (years) } & $<60$ & I & & 1 & \\
\hline & $\geq 60$ & $0.858(0.586-1.255)$ & 0.43 & $0.85(0.65 \mathrm{I}-1.109)$ & 0.231 \\
\hline \multirow[t]{2}{*}{ Sex } & Female & $\mathrm{I}$ & & 1 & \\
\hline & Male & $1.785(1.227-2.596)$ & 0.002 & $1.345(1.038-1.744)$ & 0.025 \\
\hline \multirow[t]{2}{*}{ Smoking status } & Never smoker & I & & 1 & \\
\hline & Smoker & $2.044(I .4 I-2.963)$ & $<0.0001$ & $1.409(1.082-1.833)$ & 0.011 \\
\hline \multirow[t]{5}{*}{ Location } & Right upper lobe & I & & 1 & \\
\hline & Right middle lobe & $1.009(0.519-1.959)$ & 0.98 & $1.201(0.752-1.919)$ & 0.443 \\
\hline & Right lower lobe & $0.729(0.4 I-1.296)$ & $0.28 \mathrm{I}$ & $1.018(0.688-1.506)$ & 0.929 \\
\hline & Left upper lobe & $0.828(0.5 \mathrm{II}-1.343)$ & 0.445 & $1.075(0.766-1.509)$ & 0.675 \\
\hline & Left lower lobe & $0.75(0.422-1.334)$ & 0.328 & $0.887(0.589-1.338)$ & 0.568 \\
\hline \multirow[t]{5}{*}{ Surgical procedure } & Wedge & I & & $\mathrm{I}$ & \\
\hline & Lobectomy & $\mathrm{I} .456(0.46 \mathrm{I}-4.598)$ & 0.522 & $1.796(0.739-4.367)$ & 0.196 \\
\hline & Bilobectomy & $2.626(0.733-9.417)$ & 0.138 & $3.911(1.486-10.292)$ & 0.006 \\
\hline & Sleeve lobectomy & $9.492(2.111-42.682)$ & 0.003 & $7.255(2.09-25.187)$ & 0.002 \\
\hline & Pneumonectomy & $0.959(0.1-9.235)$ & 0.971 & $6.562(2.143-20.097)$ & 0.001 \\
\hline \multirow[t]{5}{*}{ T stage } & $\mathrm{Tla}$ & 1 & & 1 & \\
\hline & TIb & $0.394(0.087-1.777)$ & 0.225 & $1.201(0.667-2.164)$ & 0.542 \\
\hline & $\mathrm{T} 2 \mathrm{a}$ & $4.168(2.218-7.835)$ & $<0.0001$ & $2.594(1.765-3.813)$ & $<0.0001$ \\
\hline & $\mathrm{T} 2 \mathrm{~b}$ & $7.942(3.585-17.595)$ & $<0.000 \mathrm{I}$ & $5.811(3.458-9.763)$ & $<0.0001$ \\
\hline & T3 & $4.38(1.52-12.628)$ & 0.006 & $4.267(2.196-8.294)$ & $<0.0001$ \\
\hline \multirow[t]{3}{*}{ Pathologic N stage } & No & 1 & & 1 & \\
\hline & NI & $2.435(1.352-4.384)$ & 0.003 & $2.126(1.408-3.209)$ & $<0.0001$ \\
\hline & N2 & $4.62(3.045-7.008)$ & $<0.0001$ & $4.115(3.094-5.473)$ & $<0.0001$ \\
\hline \multirow[t]{4}{*}{ Histology } & Lepidic predominant & I & & 1 & \\
\hline & Acinar predominant & $1.974(0.775-5.024)$ & 0.154 & $2.777(1.395-5.529)$ & 0.004 \\
\hline & Papillary predominant & $1.466(0.465-4.62)$ & 0.514 & $1.89(0.834-4.279)$ & 0.127 \\
\hline & Solid predominant & $4.582(1.8-11.663)$ & 0.001 & $6.438(3.222-12.864)$ & $<0.000$ I \\
\hline
\end{tabular}


Table 3 (Continued)

\begin{tabular}{|c|c|c|c|c|c|}
\hline \multirow[t]{2}{*}{ Variables } & \multirow[t]{2}{*}{ Comparison } & \multicolumn{2}{|c|}{ Regional disease-free survival } & \multicolumn{2}{|c|}{ Distant disease-free survival } \\
\hline & & HR (95\% CI) & P-value & HR (95\% CI) & P-value \\
\hline & Micropapillary predominant & $5.85(2.18-15.695)$ & $<0.0001$ & $6.191(2.94-13.036)$ & $<0.000$ I \\
\hline & Mucinous predominant & $2.854(0.87-9.366)$ & 0.084 & $3.034(1.255-7.335)$ & 0.014 \\
\hline \multirow[t]{3}{*}{ Differentiation } & Well differentiated & I & & I & \\
\hline & Moderately differentiated & $3.02(1.377-6.623)$ & 0.006 & $2.346(1.49-3.693)$ & $<0.0001$ \\
\hline & Poorly differentiated & $6.766(3.055-14.983)$ & $<0.0001$ & $3.817(2.375-6.134)$ & $<0.000$ I \\
\hline \multirow[t]{2}{*}{ VPI } & Negative & I & & I & \\
\hline & Positive & $2.34 \mathrm{I}(1.564-3.504)$ & $<0.000 \mathrm{I}$ & $2.193(1.665-2.89)$ & $<0.0001$ \\
\hline \multirow[t]{2}{*}{ LVI } & Negative & I & & I & \\
\hline & Positive & $2.146(1.437-3.205)$ & $<0.0001$ & $2.415(1.823-3.2)$ & $<0.0001$ \\
\hline
\end{tabular}

Abbreviations: $\mathrm{Cl}$, confidence interval; HR, hazard ratio; LVI, lymphovascular space invasion; VPI, visceral pleural invasion.

We performed multivariate analysis to determine independent prognostic and predictive factors for DFS, OS, regional recurrence, and distant metastasis using Cox forward stepwise regression model (Table 4). Advanced T stage, lymph node metastasis, and solid and micropapillary predominant histology were found to be independent prognostic factors for OS and DFS.

Advanced T stage (T1b-T2b), lymph node metastasis (N2), and poor differentiation were found to be significant adverse predictive factors for regional disease recurrence. No significant difference in regional recurrence was observed in terms of sex, smoking history, surgical procedures, predominant histology, lymphovascular invasion, or VPI in multivariate analysis (Table 4).

T2b stage, lymph node metastasis (N1-2), and solid and micropapillary predominant histology were significant factors for predicting distant disease recurrence. VPI was associated with distant recurrence with a marginal significance $(P=0.089)$. No significant correlation was seen between distant failure and sex, smoking history, surgical procedures, or lymphovascular invasion in multivariate analysis (Table 4).

In patients who had pleural invasion, solid or micropapillary histology was significantly associated with poor DFS and OS, in comparison with those without (Figure 1). Since VPI was found to be correlated with solid and micropapillary predominant histology, we further compared the survival rates between VPI-positive and VPI-negative patients grouped by histologic subtypes. In patients with VPI, significantly shorter DFS and OS were seen in patients with solid or micropapillary predominant histology than in patients with lepidic, acinar, papillary, or mucinous adenocarcinomas, regardless of stage (Figure 2). For patients with stage I or II disease, positive VPI and relatively favorable predominant growth pattern (lepidic, acinar, and papillary growth patterns) significantly correlated to long DFS, which was not found in patients with stage III disease. There was no significant difference in OS between VPI-negative and -positive patients with relative favorable predominant growth pattern regardless of stage (Figure 2).

In the seventh TNM staging system, a tumor $<3 \mathrm{~cm}$ with VPI is classified as T2a and VPI-positive patient with stage Ib might benefit from adjuvant chemotherapy. Among the 95 stage Ib patients with VPI, no significant differences were observed in DFS and OS between patients who received adjuvant chemotherapy and those who did not (Figure S2). Of the 95 patients with stage Ib, 22 patients who had solid predominant tumor were further selected for analyzing the impact of adjuvant chemotherapy on DFS and OS. As shown in Figure 3, patients who received adjuvant chemotherapy had longer DFS and OS than the patients who received no chemotherapy after surgery. However, these differences in PFS and OS did not reach statistical significance.

\section{Discussion}

In the present study, we investigated the impact of VPI status on survival according to the new IASLC/ATS/ERS classification of lung adenocarcinoma and analyzed the predictive and prognostic values of VPI. The new IASLC/ATS/ERS classification system of lung adenocarcinoma is an efficient discriminator for prognosis of patients. ${ }^{10-14}$ Patients with NSCLC with micropapillary or solid growth pattern have significantly poor OS and DFS, in comparison to other growth patterns. ${ }^{10,11}$ Our results also demonstrated that micropapillary and solid predominant histology had poor OS and was associated with early distant failure after surgery. Previous studies showed that visceral pleural involvement is an independent factor of poor prognosis in patients with NSCLC. ${ }^{2-4}$ However, the underlying reason still remains unclear. Our present study demonstrated that VPI was a significant prognostic and predictive factor for DFS and OS, independently of sex, smoking status, surgical procedures, stage, tumor grade, and 


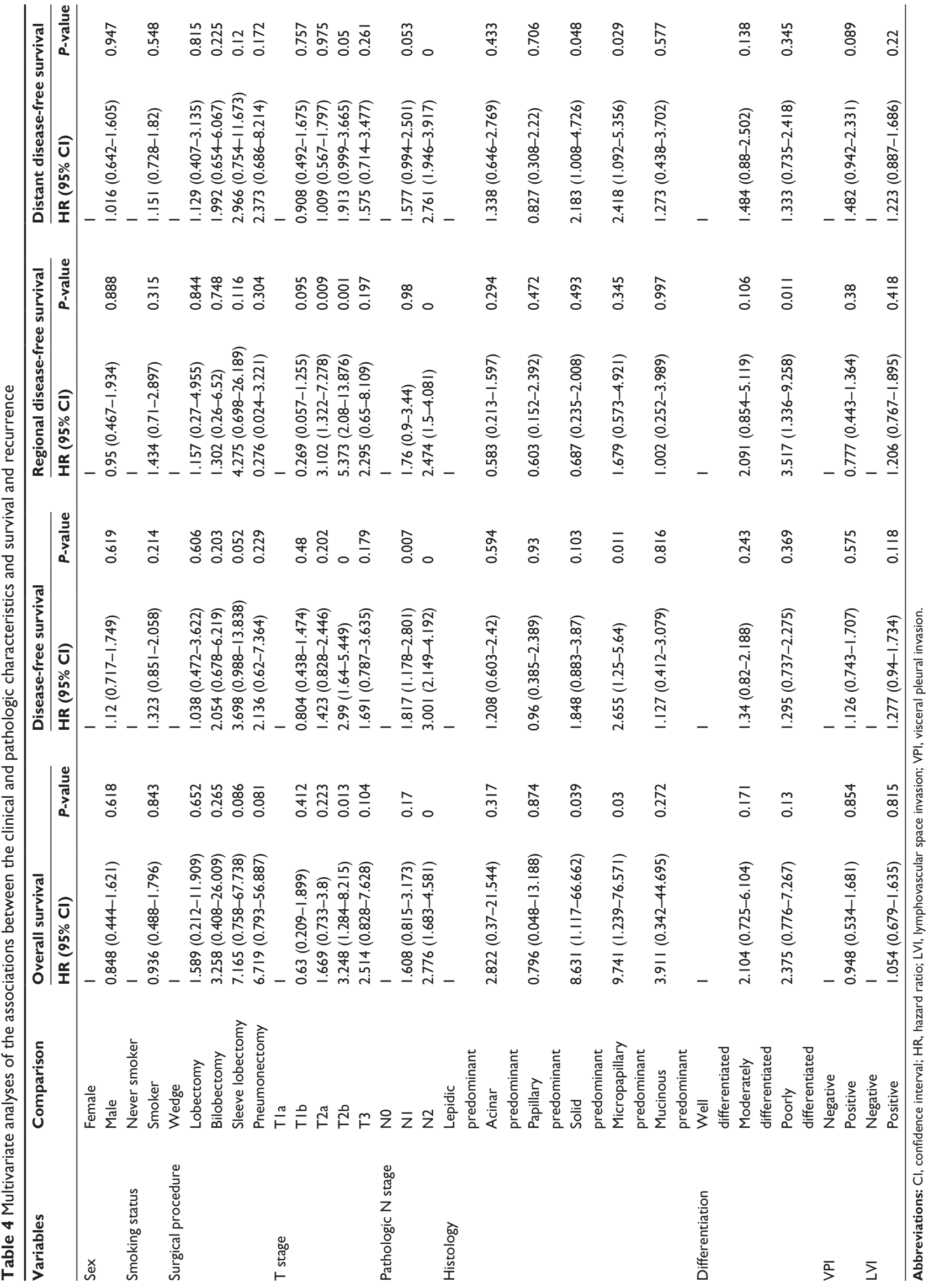



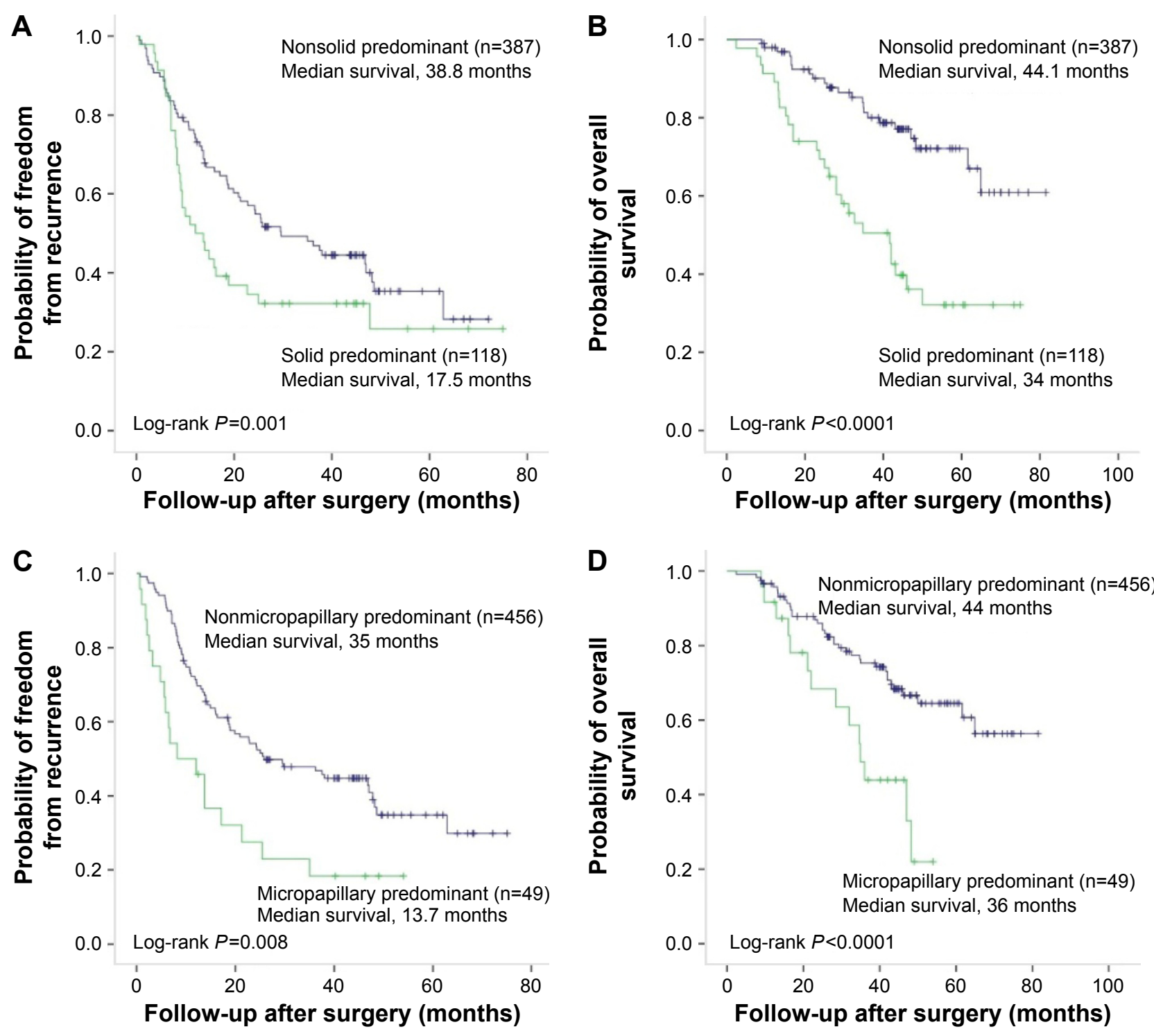

Figure I Kaplan-Meier survival curves for disease-free survival (A, C) and overall survival (B, D) according to predominant growth patterns in 266 patients with lung adenocarcinoma with visceral pleural invasion.
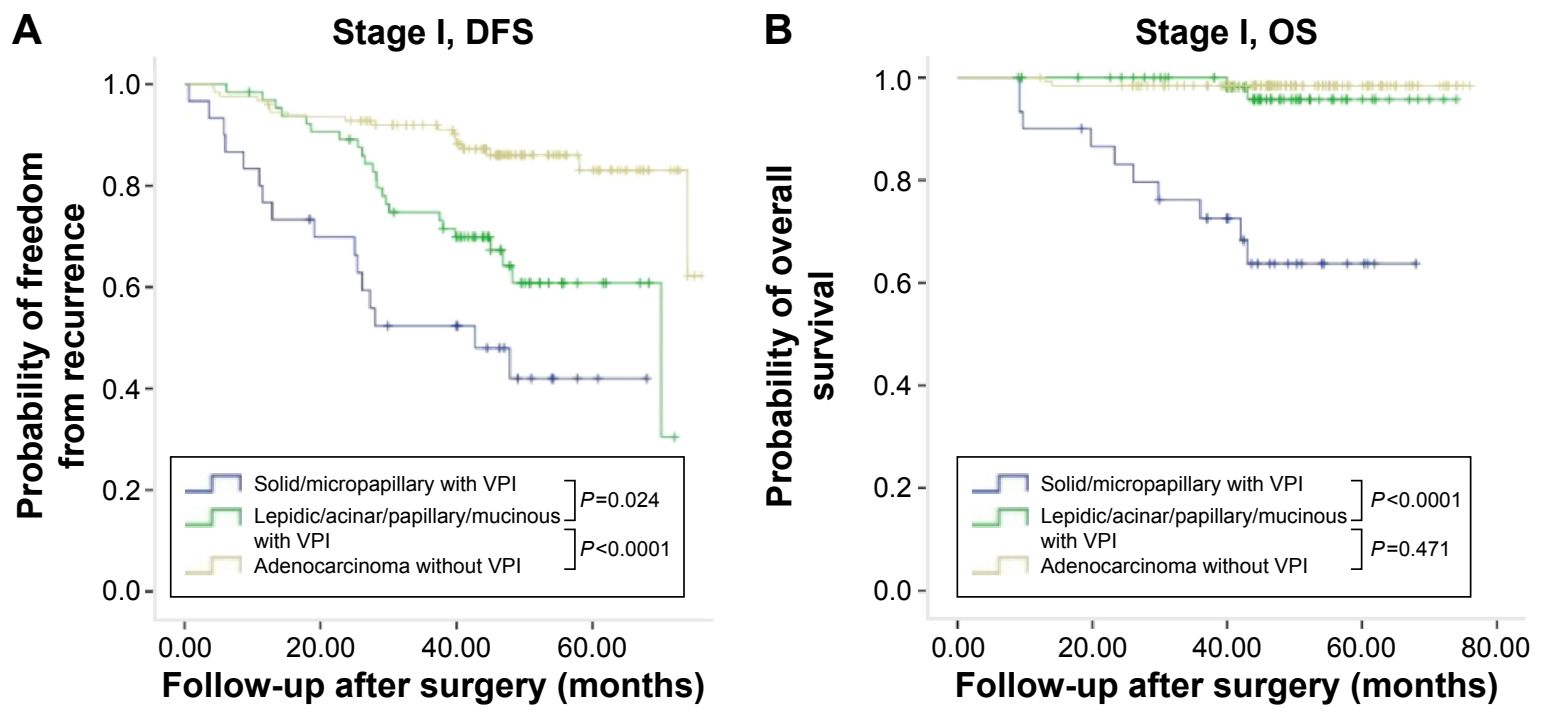

Figure 2 (Continued) 

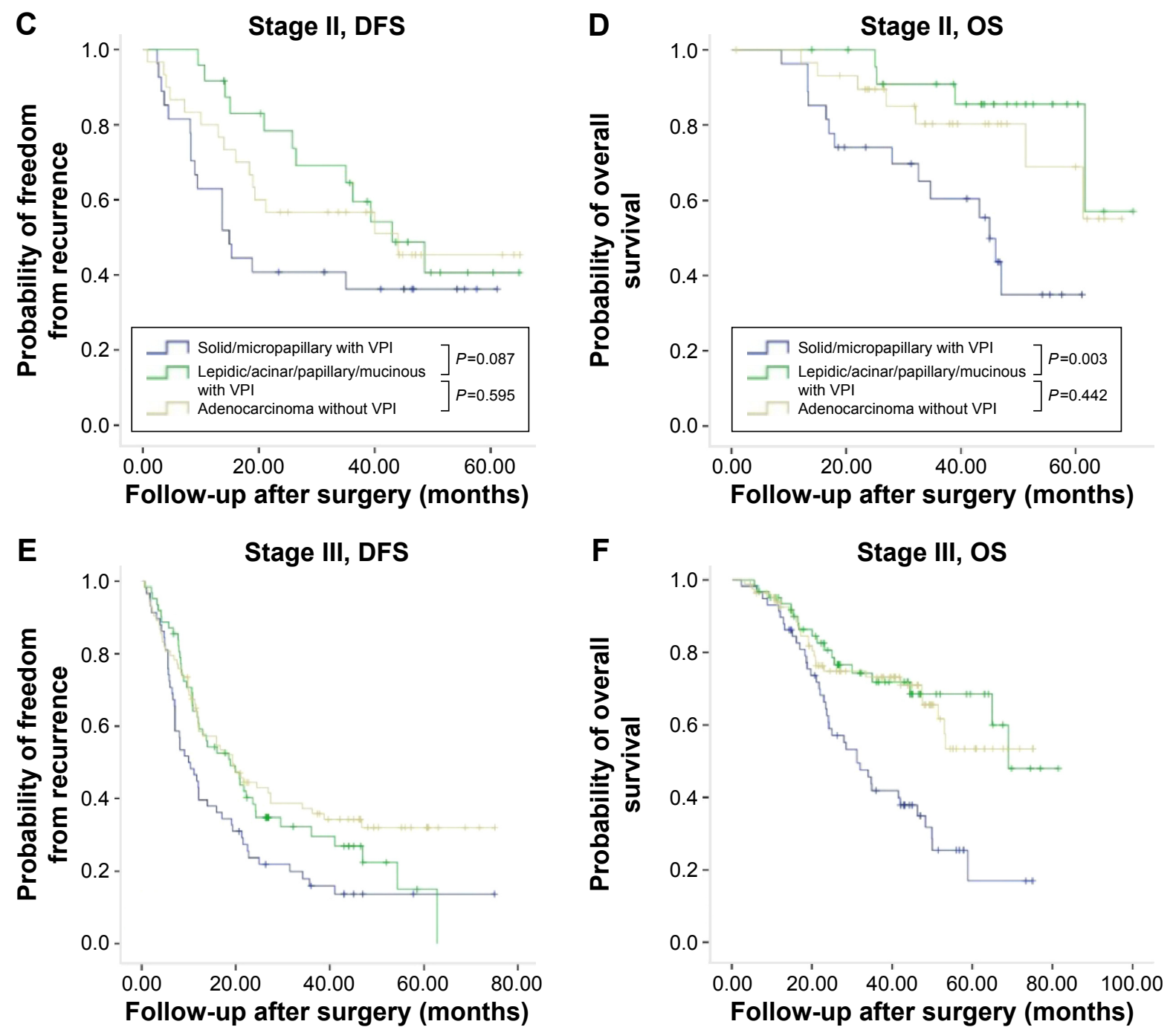

Follow-up after surgery (months)

Follow-up after surgery (months)

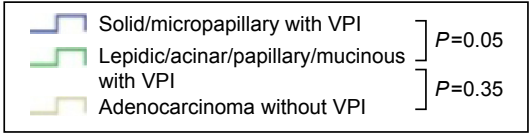

$\left.\begin{array}{l}\square \text { Solid/micropapillary with VPI } \\ \left.\begin{array}{l}\text { Lepidic/acinar/papillary/mucinous } \\ \text { with VPI }\end{array}\right] P<0.0001 \\ \text { Adenocarcinoma without VPI }\end{array}\right] P=0.657$

Figure 2 Kaplan-Meier survival curves for DFS and OS by predominant growth patterns and pathological TNM stage in 266 patients with lung adenocarcinoma with VPI. Notes: (A) Cumulative probability of recurrence-free survival in patients with stage I disease stratified by predominant histology and VPI. (B) Cumulative probability of OS in patients with stage I disease stratified by predominant histology and VPI. (C) Cumulative probability of recurrence-free survival in patients with stage II disease stratified by predominant histology and VPI. (D) Cumulative probability of OS in patients with stage II disease stratified by predominant histology and VPI. (E) Cumulative probability of recurrence-free survival in patients with stage III disease stratified by predominant histology and VPI. (F) Cumulative probability of OS in patients with stage III disease stratified by predominant histology and VPI.

Abbreviations: DFS, disease-free survival; OS, overall survival; VPI, visceral pleural invasion.

lymphovascular space invasion, which was consistent with previous studies..$^{2-4} \mathrm{We}$ also demonstrated that the VPI was frequently found in patients with solid or micropapillary histology and less frequently found in patients with lepidic predominant adenocarcinoma. The multivariate analysis showed that VPI was not an independent predictor for OS, DFS, and disease recurrence after adjustment for the new classification system of lung adenocarcinoma. Solid predominant histology and micropapillary predominant histology were predictive factors for OS and DFS, independent of stage. Given these findings, the prognostic predictive value of VPI might be dependent on the histology subtype of lung adenocarcinoma and explains the inconsistency of previous reports. ${ }^{2-4,6-8}$

Identifying patients with high postoperative recurrence risk could guide adjuvant-individualized therapies. The risk factors of recurrence for resected lung adenocarcinoma have not been well established. TNM staging was the strongest known predictive factor for regional and distant disease recurrence in resected NSCLCs. ${ }^{10,11}$ We also confirmed that advanced $\mathrm{T}$ stage and lymph node metastasis were independent predictive factors for regional and distant recurrence in 

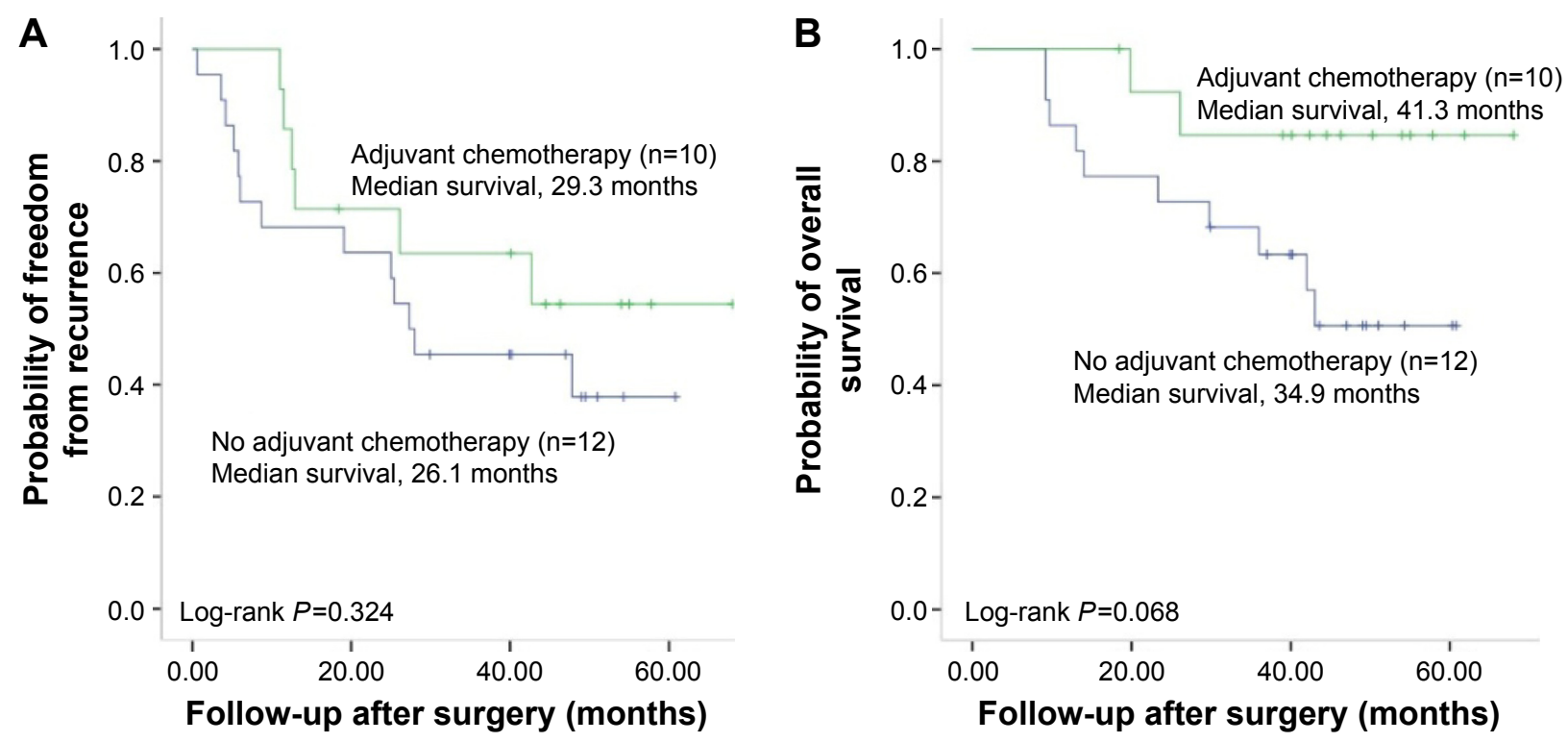

Figure 3 Kaplan-Meier survival curves for disease-free survival (A) and overall survival (B) by adjuvant chemotherapy in 22 solid predominant lung adenocarcinomas with visceral pleural invasion.

surgically resected adenocarcinomas. However, there is no consensus on whether VPI was an independent risk factor for regional and distant recurrence. Multivariate analysis showed that VPI was associated with both regional and distant disease recurrence in lung adenocarcinomas but was not an independent predictive factor. It should be noted that poor differentiation was an independent predictive factor for regional recurrence and solid/micropapillary predominant histology was a stage-independent predictor for distant recurrence in this patient cohort.

Currently, adjuvant chemotherapy is not included in the standard treatment for stage Ib NSCLC and its role in stage $\mathrm{Ib}$ disease remains controversial. ${ }^{15}$ There is no reliable marker available to predict the effectiveness of chemotherapy. Previous studies found that chemotherapy could reduce the survival of patients with lepidic predominant adenocarcinoma. ${ }^{16,17}$ In contrast, solid predominant adenocarcinoma responded well to adjuvant chemotherapy. ${ }^{10,11}$ Tumor cells might disseminate from original primary tumor before surgery and result in distant recurrence in patients with VPI. Some clinicians are inclined to apply systemic adjuvant chemotherapy in stage Ib NSCLC with VPI. ${ }^{18}$ Therefore, it is necessary to identify the subgroup of stage Ib patients who could benefit from adjuvant chemotherapy. We retrospectively reviewed patients with stage $\mathrm{Ib}$ disease and found that a total of 108 (21.4\% of 505) patients with adenocarcinoma had stage $\mathrm{Ib}$ disease and $88 \%(95 / 108)$ of the patients had VPI. Among the 95 patients, there was no significant difference in DFS and OS between patients who received adjuvant chemotherapy and those who did not. Taken together with previous studies, ${ }^{19,20}$ VPI might not be a useful factor to determine whether patients with stage $\mathrm{Ib}$ disease should receive adjuvant chemotherapy. We also noticed that stage Ib patients with solid predominant growth pattern and who received adjuvant chemotherapy had longer DFS and OS than those who did not, but without statistical significance. However, because the present study included only 22 stage Ib patients with solid growth pattern, this finding should be cautiously interpreted and validated in future well-designed prospective trials.

We acknowledge that the study has some limitations. Elastic staining was not performed in most of the included cases. Elastic staining can make it much easier for the pathologists to identify the extent of pleural invasion. There is no consensus on whether elastic staining should be routinely performed in VPI evaluation. The Japan Lung Cancer Society recommends that both H\&E staining and elastic staining should be used to assess VPI. ${ }^{21}$ Since elastic staining is unnecessary for most cases to evaluate the pleura for invasion, the IASLC recommends using elastic stains only when the distinction between PL0 and PL1 is not clear based on evaluation of H\&E sections. ${ }^{12}$ In the present study, we defined VPI as invasion of tumor beyond the elastic layer (PL1) or to the pleural surface (PL2), as the IASLC suggested. ${ }^{12}$ In some cases where the distinction of visceral pleura elastic fibers was difficult or the tumor cells were very close to the visceral pleural margin, we applied elastic van Gieson stains in VPI evaluation. Since elastic staining was not routinely 
performed, it is possible that a few tumors invading to the visceral pleural might be misdiagnosed.

\section{Conclusion}

We demonstrated that VPI was associated with solid and micropapillary histology, which might be the underlying reason why tumors with VPI have an unfavorable prognosis. In addition, stage $\mathrm{Ib}$ patients with solid histologic subtype might benefit from adjuvant chemotherapy, which warrants further clinical randomized trials for validation.

\section{Disclosure}

The authors report no conflicts of interest in this work.

\section{References}

1. Jemal A, Bray F, Center MM, Ferlay J, Ward E, Forman D. Global cancer statistics. CA Cancer J Clin. 2011;61:69-90.

2. Chang YL, Lin MW, Shih JY, Wu CT, Lee YC. The significance of visceral pleural surface invasion in 321 cases of non-small cell lung cancers with pleural retraction. Ann Surg Oncol. 2012;19:3057-3064.

3. Neri S, Yoshida J, Ishii G, et al. Prognostic impact of microscopic vessel invasion and visceral pleural invasion in non-small cell lung cancer. Ann Surg. 2014;260:383-388.

4. Hung JJ, Jeng WJ, Hsu WH, Chou TY, Lin SF, Wu YC. Prognostic significance of the extent of visceral pleural invasion in completely resected node-negative non-small cell lung cancer. Chest. 2012;142:141-150.

5. Goldstraw P, Crowley J, Chansky K, et al. The IASLC Lung Cancer Staging Project: proposals for the revision of the TNM stage groupings in the forthcoming (seventh) edition of the TNM Classification of malignant tumours. J Thorac Oncol. 2007;2:706-714.

6. Lopez Guerra JL, Gomez DR, Lin SH, et al. Risk factors for local and regional recurrence in patients with resected N0-N1 non-small-cell lung cancer, with implications for patient selection for adjuvant radiation therapy. Ann Oncol. 2013;24:67-74.

7. Ou SH, Zell JA, Ziogas A, Anton-Culver H. Prognostic significance of the non-size-based AJCC T2 descriptors: visceral pleura invasion, hilar atelectasis, or obstructive pneumonitis in stage IB non-small cell lung cancer is dependent on tumor size. Chest. 2008;133:662-669.

8. Hung JJ, Jeng WJ, Hsu WH, Chou TY, Huang BS, Wu YC. Predictors of death, local recurrence, and distant metastasis in completely resected pathological stage-I non-small-cell lung cancer. J Thorac Oncol. 2012;7: 1115-1123.

9. Travis WD, Brambilla E, Noguchi M, Hirsch FR; IASLC Pathology Committee. International Association for the Study of Lung Cancer/ American Thoracic Society/European Respiratory Society International Multidisciplinary Classification of Lung Adenocarcinoma. J Thorac Oncol. 2011;6:244-285.
10. Hung JJ, Yeh YC, Jeng WJ, et al. Predictive Value of the International Association for the Study of Lung Cancer/American Thoracic Society/ European Respiratory Society classification of lung adenocarcinoma in tumor recurrence and patient survival. J Clin Oncol. 2014;32: 2357-2364.

11. Warth A, Muley T, Meister M, et al. The novel histologic International Association for the Study of Lung Cancer/American Thoracic Society/ European Respiratory Society Classification System of lung adenocarcinoma is a stage-independent predictor of survival. J Clin Oncol. 2012;30:1438-1446.

12. Travis WD, Brambilla E, Rami-Porta R, et al. Visceral pleural invasion: pathologic criteria and use of elastic stains: proposal for the 7th edition of the TNM classification for lung cancer. J Thorac Oncol. 2008;3: 1384-1390.

13. Tsuta K, Kawago M, Inoue E, et al. The utility of the proposed IASLC/ ATS/ERS lung adenocarcinoma subtypes for disease prognosis and correlation of driver gene alterations. Lung Cancer. 2013;81: 371-376.

14. Mansuet-Lupo A, Bobbio A, Blons H, et al. The new histologic classification of lung primary adenocarcinoma subtypes is a reliable prognostic marker and identifies tumors with different mutation status: the experience of a French cohort. Chest. 2014;146:633-643.

15. Geiger S, Schlemmer M, Heinemann V, Stemmler HJ. Adjuvant cisplatin-based chemotherapy for resected NSCLC: one size fits all? Anticancer Drugs. 2010;21:799-804.

16. Miller VA, Hirsch FR, Johnson DH. Systemic therapy of advanced bronchioloalveolar cell carcinoma: challenges and opportunities. J Clin Oncol. 2005;23:3288-3293.

17. Schiller JH1, Harrington D, Belani CP, et al. Comparison of four chemotherapy regimens for advanced non-small-cell lung cancer. $N$ Engl J Med. 2002;346:92-98.

18. Maeda R, Yoshida J, Ishii G, Hishida T, Nishimura M, Nagai K. Poor prognostic factors in patients with stage IB non-small cell lung cancer according to the seventh edition TNM classification. Chest. 2011;139: 855-861.

19. Oven Ustaalioglu BB, Unal OU, Turan N, et al. Prognostic factors for lymph node negative stage I and IIA non-small cell lung cancer: multicenter experiences. Asian Pac J Cancer Prev. 2013;14:6287-6292.

20. Nitadori J, Colovos C, Kadota K, et al. Visceral pleural invasion does not affect recurrence or overall survival among patients with lung adenocarcinoma $\leq 2 \mathrm{~cm}$ : a proposal to reclassify $\mathrm{T} 1$ lung adenocarcinoma. Chest. 2013;144:1622-1631.

21. Shimizu K, Yoshida J, Nagai K, et al. Visceral pleural invasion classification in non-small cell lung cancer: A proposal on the basis of outcome assessment. J Thorac Cardiovasc Surg. 2004;127:1574-1578. 


\section{Supplementary materials}

A

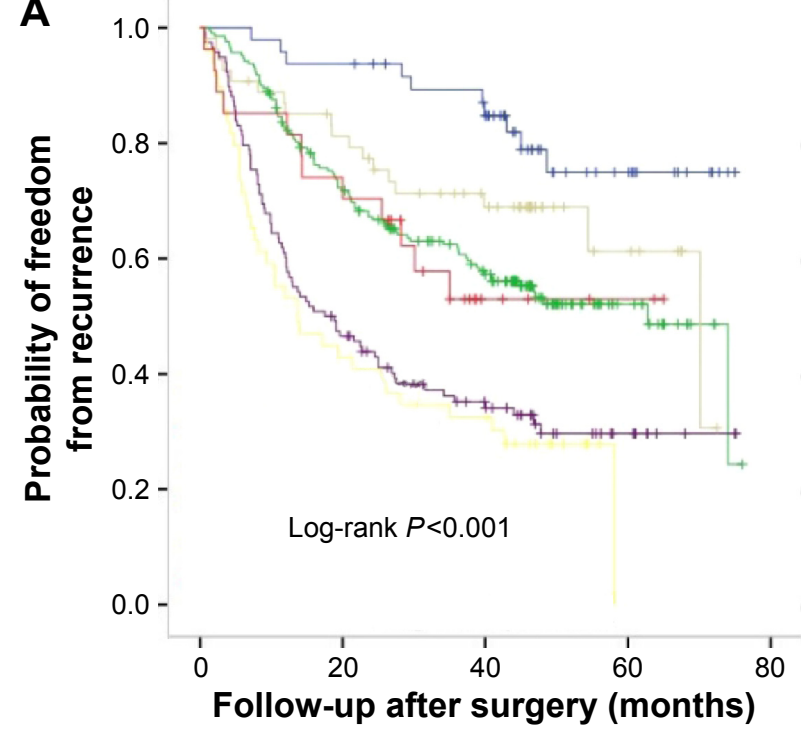

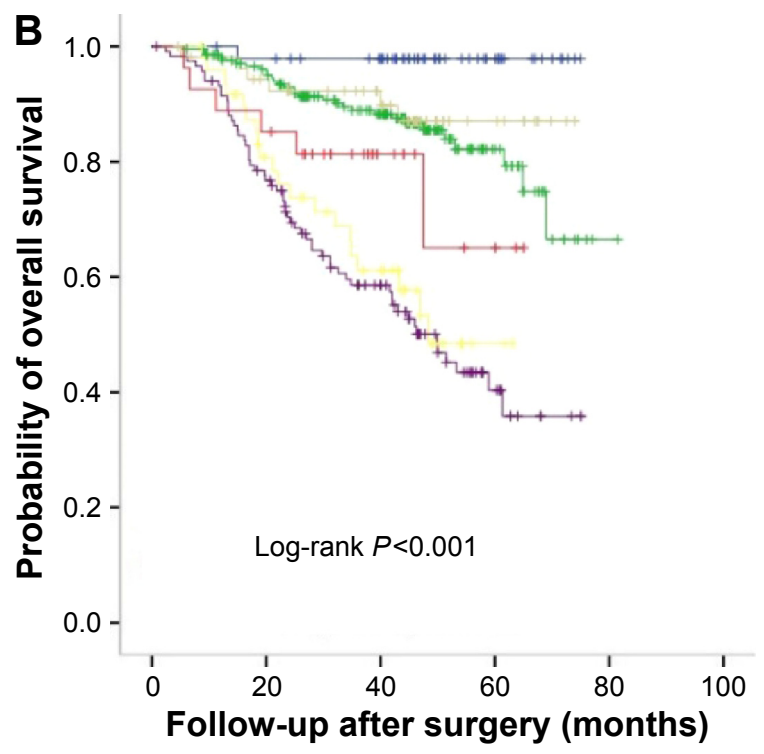

Follow-up after surgery (months)

\begin{tabular}{|c|c|c|}
\hline$\neg$ Lepidic $(n=49)$ & $\neg$ Acinar $(n=207)$ & $\square$ Papillary $(n=53)$ \\
\hline$\neg$ Solid $(n=118)$ & Micropapillary $(n=49)$ & $\neg$ Mucinous $(n=27)$ \\
\hline
\end{tabular}

Figure SI Kaplan-Meier survival curves for disease-free survival (A) and overall survival (B) according to predominant growth patterns in 505 patients with lung adenocarcinoma.

A

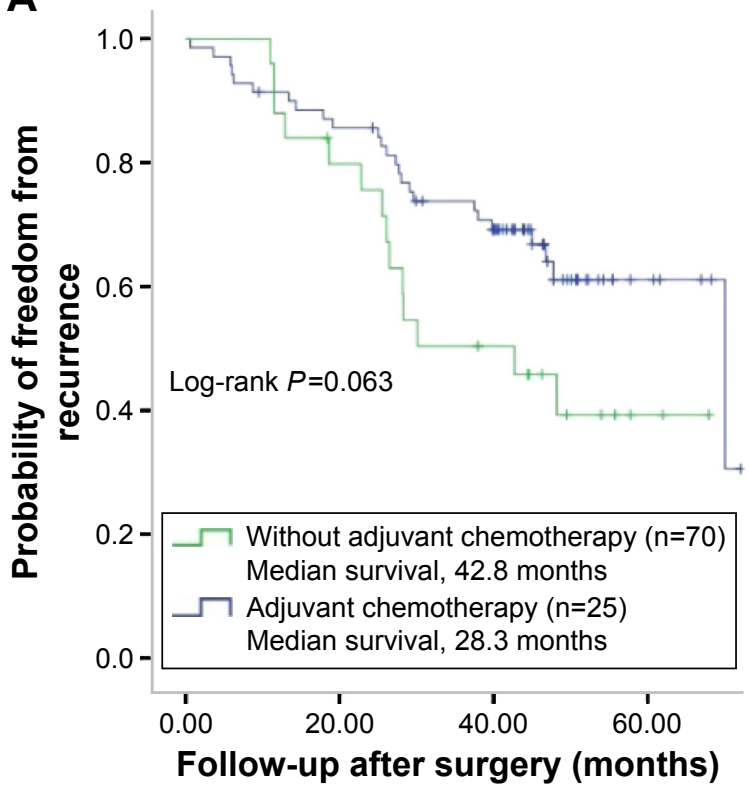

B

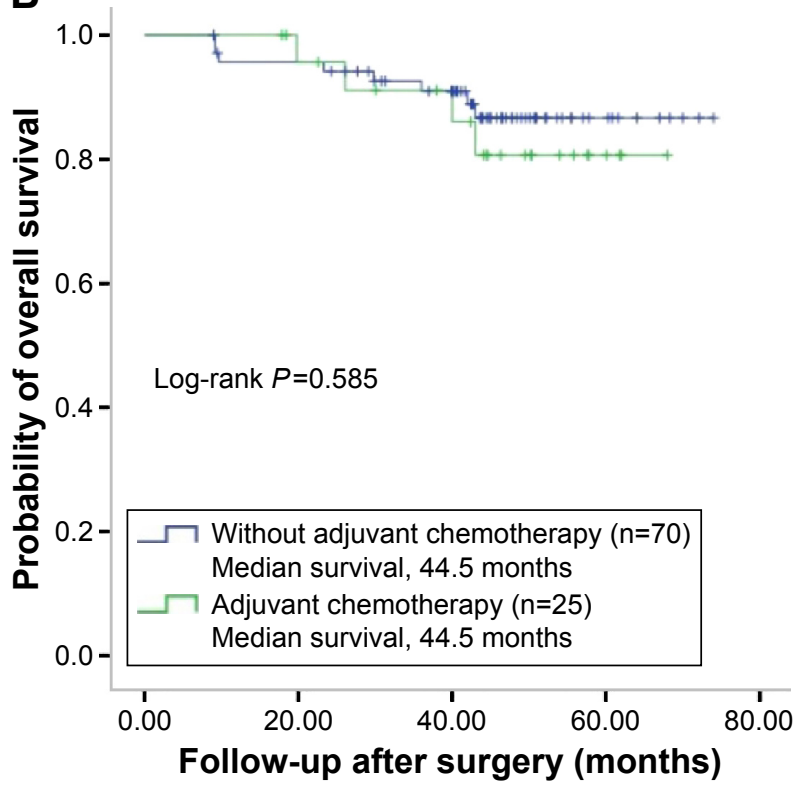

Figure S2 Kaplan-Meier survival curves for disease-free survival (A) and overall survival (B) according to adjuvant chemotherapy in 95 stage lb patients with lung adenocarcinoma with visceral pleural invasion.

\section{Publish your work in this journal}

OncoTargets and Therapy is an international, peer-reviewed, open access journal focusing on the pathological basis of all cancers, potential targets for therapy and treatment protocols employed to improve the management of cancer patients. The journal also focuses on the impact of management programs and new therapeutic agents and protocols on patient perspectives such as quality of life, adherence and satisfaction. The manuscript management system is completely online and includes a very quick and fair peer-review system, which is all easy to use. Visit http://www.dovepress.com/testimonials.php to read real quotes from published authors. 